\title{
Premature Mortality due to Breast Cancer in a Mountainous Province of Vietnam from 2005 to 2018
}

\author{
Kim Cat Tuyen Vo ${ }^{1 *}$ and Ngoan Tran Le $\mathrm{Le}^{2,3}$ \\ ${ }^{1}$ Graduate School of Medicine, International University of Health and Welfare, Japan \\ ${ }^{2}$ Institute of Research and Development, Duy Tan University, Vietnam \\ ${ }^{3}$ Department of Public Health, School of Medicine, International University of Health and Welfare, Japan \\ *Corresponding author: Kim Cat Tuyen Vo, Graduate School of Medicine, International University of Health and Welfare, Narita, \\ Japan
}

\begin{abstract}
ARTICLE INFO
Received: 㓞 December 17, 2021

Published: 幽 January 11, 2022

Citation: Kim Cat Tuyen Vo, Ngoan Tran Le. Premature Mortality due to Breast Cancer in a Mountainous Province of Vietnam from 2005 to 2018. Biomed J Sci \& Tech Res 40(5)-2022. BJSTR. MS.ID.006525.
\end{abstract}

Keywords: Breast Cancer; Cancer-Mortality; Premature-Mortality; Developing Countries; Vietnam

Abbreviations: CDC: Center of Diseases Control; ASR: Age-Standardized Mortality Rates; GNI: Gross National Income

\section{ABSTRACT}

Objective: There is limited information on breast cancer in remote areas in Vietnam. This study aimed to identify the mortality of breast cancer among women in Lang Son mountainous province to inform local as well as nationwide control strategies of the disease.

Methods: This is a population-based study conducted at the state commune health stations of Lang Son province, recording deaths from breast cancer during 2005-2018. We derived case variables of ID, age, sex, date of death, and cause of death from the mortality database of Lang Son Center of Diseases Control (CDC). The included cases were the women diagnosed and died of breast cancer. Age-standardized mortality rates per 100,000 (ASR) and mortality rates ratio and 95\% confidence interval (MRR, $95 \% \mathrm{CI})$ were estimated.

Results: From January 2005 to December 2018, there were 210 decedents out of 17,990 (1.17\% of total mortality) women diagnosed with breast cancer in Lang Son province. World population structure (2000-2025) age-standardized mortality rate was 5.2 per 100,000. Compared to the period 2005-2006, non-significant increase risk was observed in 2018, age-adjusted MRR, 95\% CI: 1.36, 0.78, 2.35, p for trend 0.209 . The proportion of premature death under the age of 70 was $81.4 \%$.

Conclusion: The present study highlights the importance of population education to screen for breast cancer to prevent young age death in remote areas. It is also critical to plan the strategy for improving the competence of diagnostic and treatment for local hospitals to prevent premature death due to this preventable disease.

\section{Introduction}

Breast cancer has been worldwide recognized to have a high mortality rate among women despite wide variations in ethnicity, culture, and economics. It was reported that breast cancer is the most commonly diagnosed cancer among women in 140 of 184 countries worldwide [1]. More than one million new cases of female breast cancer are diagnosed each year. Approximately one in 4 of all new cancer cases diagnosed in women worldwide are breast cancer, followed by $6.6 \%$ of cancer death in 2018 [2]. The breast cancer mortality rate has been substantially increasing in the world during the past 25 years [3]. According to the American Cancer Society, the overall breast cancer incidence rates increased among Asian/ Pacific Islander (1.7\% per year), non-Hispanic back $(0.4 \%$ per year), and Hispanic (0.3\% per year) [4]. In China, mortality from 
breast cancer rose progressively during the past three decades in both rural and urban areas [5]. An estimated 268600 women were diagnosed and 69500 died of breast cancer in 2015, accounting for $15.1 \%$ of all new cancer cases and $6.9 \%$ of all cancer deaths in Chinese women, respectively [6].

Vietnam, situated in Southeast Asia, is a developing country with a GNP per capita of USD 2,540 in 2019. Social health insurance is voluntary of approximately US $\$ 20.00$ per annum, which is paid by individuals and their households. In Vietnam, breast cancer is reported to be the most common cancer in Vietnamese women [7]. There were 15,229 cases of breast cancer were diagnosed in 2018, accounting for $5.3 \%$ of all cancer deaths [2]. In response to this situation, the Vietnamese government has introduced nationwide breast self-examination education as the method for early detection [8]. Moreover, the usage of mammography and hormone therapy for patients with estrogen-receptor-positive breast cancers has been improved the life expectancy of patients with breast cancer [9]. However, lacking population-based studies about this fatal disease hindered the establishment of focused primary prevention strategies. The mortality rate is a basic and critical indicator for the development of appropriate and effective intervention programs and monitoring the health of patients with breast cancer. Lang Son is a mountainous province in the Northeast region with a natural area of 8,187.25 km², bordering Guangxi province in China.

The population of the province in 2019 is 782,811 people, mainly living in rural areas (accounting for 80.7\%). Lang has a high proportion of ethnic minorities $(84.74 \%$ of the province's population). Currently, there are 30,583 poor households, accounting for 15.83\%; 21,267 near-poor households, accounting for $11.01 \%$ [10]. Having these geographic economic conditions, Lang Son province is considered as the representative of mountainous provinces in Vietnam where the citizens have a limited chance to approach high-quality health care services. In this context, this study was conducted to clarify the status of breast cancer mortality in Lang Son province from January 2005 to December 2018 to provide an updated and comprehensive understanding of recent trends of breast cancer mortality in this province.

\section{Material and Methods}

\section{Study Design and Data Source}

This is a population-based study of the time series of deaths from breast cancer of women living in Lang Son province. The data was collected in two steps. At first, the mortality information was recorded in an official book referred to by The Ministry of Health's death recording systems (A6) managed by the Lang Son CDC. The A6 mortality systems were validated and presented to be a reliable and feasible system for mortality recording [11]. These unique systems were introduced to be used nationwide in Vietnam in 1992. All deaths occurring in the communities were registered at the state commune health stations. The data from the A6 was collated and determined monthly by the head of the state commune health stations who in turn forward the information every year to the Lang Son CDC from 2005 to date to develop a database of mortality there. Five indicators included the case's ID, age, sex, date - place - cause of death, and ICD-10 coding. To prevent duplicate records, the head of the commune health station, trained medical workers, followed carefully the medical care for each morbidity case for each resident for at least 6 months until the outcome was identified. By this follow-up process, all deaths in the list have described the cause of death based on medical records. Besides, data on population was collected with careful cross-check with several independent information sources such as the departments for provincial statistics, the department of Population - Family - Children, the Committee of Family and Planning. We included all cases that were dead of breast cancer from January 2005 to December 2018, ICD-10: C50 for the present study. The Lang Son population-based mortality registration covered over 226 state commune health stations of 11 cities/districts of the province. The average resident number of each state commune health station was annually collected by the Lang Son CDC.

\section{Data Analysis}

Data was reviewed and cross-checked between information sources, cleaned, encoded, and reported by Excel software, analyzed by STATA 15.0. For the calculation of the mortality rates of breast cancer, the ratio was determined between the number of deaths from breast cancer in women and the female population in that year, multiplied by 100,000 . The world population structure and the Segi's world population standard were used to estimate agestandardized mortality rates per 100,000 (ASR). Mortality rates ratio and $95 \%$ confidence interval adjusted for age groups $(0-9,10$ $19,20-29,30-39,40-49,50-59,60-69,70-79,80+$ ) was estimated to observe time trend from 2005 to 2018.

\section{Ethical Approval}

This is a population-based study about mortality rate with no interfere with no physical intervention. This information to identify patients including name, personal address, date of birth, would be not published. Moreover, due to the database purpose, which is to provide accessible data to help improve the mortality of breast cancer in the community, the public interest is considered to outweigh personal interests in privacy or autonomy that would otherwise be protected by consent mechanisms. The research protocol was approved by the Hanoi Medical University Review Board in Bio-Medical Research \# 61/HMURB, dated 25 November 2008 and by the Board of Ethics in Bio-Medical Research at 
University of Medicine and Pharmacy at Ho Chi Minh City \#106/ UMP-BOARD, dated 20 March 2019.

\section{Results}

From January 2005 to December 2018 (missing data for 20092010), there were 210 deaths out of 17,990 women diagnosed with breast cancer in Lang Son province. Table 1 showed the crude mortality rate and the age-standardized mortality rates from breast cancer at all ages between 2005 and 2018. The crude mortality rate of breast cancer was 4.7. After adjusting using the World Health Organization standard population for 2000 - 2025 and the Segi World standard population (in the 1960s), the ASR was 5.2 and 4.7, respectively. Over four-fifths of death cases died under the age of 70 (81.5\%). In Table 2, the crude rate and the ASR rate were annually calculated. The lowest ASR per 100,000 person-year was found in 2006 (3.2). The value of 7.0 was the highest ASR that occurred in 2012. When the first two-year 2005-2006 was the reference group, the mortality rate ratio for 2018 was increased, but not statistically significant, age-adjusted MRR, 95\%CI: 1.36, 0.78, 2.35, $\mathrm{p}$ for trend=0.209, (Table 3). Figure 1 presented the age-specific mortality rate per 100,000 person-years during the study period. The mortality rate was found to be at a low and stable level from the age group 0-9 to the age group 20-29. There was, on the other hand, a rapid rate of increase at the reproductive age, especially at the age group 40 - 59, and slow down after that. The highest specific cancer mortality rates per 100,000 were seen for the age group $60-69$. The curve thereafter was seen to be declined (Figure 2).

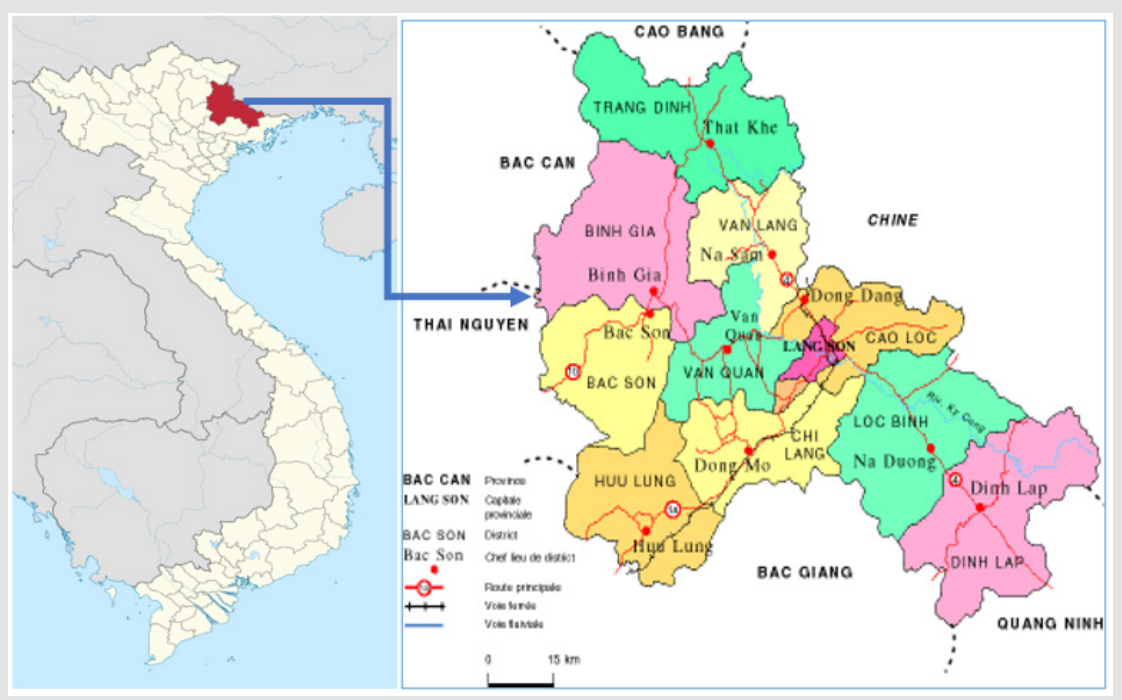

Figure 1: Location of the study population, Lang Son province in Viet Nam.

(Source: https:/ / commons.wikimedia.org/wiki/File:Lang_Son_in_Vietnam.svg )

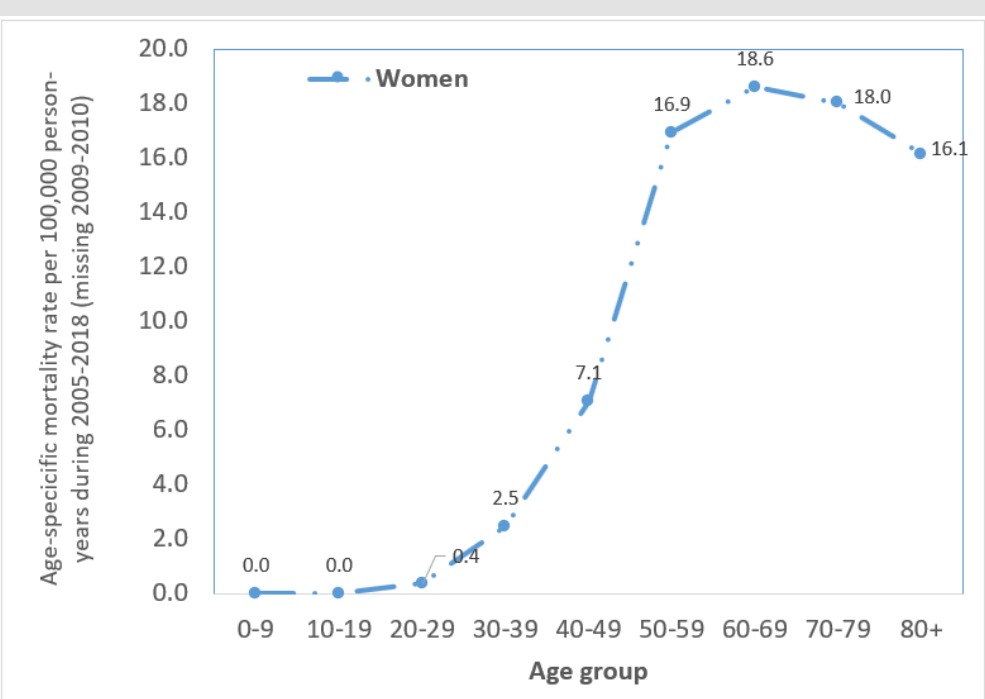

Figure 2: Age-specific mortality rate per 100,000 person-years in women during 2005-2018 (missing data for 2009-2010) due to breast cancer 
Table 1: Mortality due to breast cancer in women during 2005-2018 in Lang Son province \& Crude rate per 100,000 person-years; @ Age-standardized rate per 100,000 person-years using the World Health Organization standard population for 2000-2025; \# Proportion of death cases aged under 70 year-olds. @ Age-standardized rate per 100,000 person-years using SEGI World standard population (in the 1960s). Missing data for 2009-2010.

\begin{tabular}{|c|c|c|c|c|c|c|c|}
\hline Sex & Year & ICD-10 & Total Death & Crude rate \& & ASR @ & \% $<70$ \# & WHO \$ \\
\hline Women & $2005-18$ & C50 & 210 & 4.6 & 4.7 & 81.4 & 5.2 \\
\hline
\end{tabular}

Table 2: Mortality due to breast cancer in women by year from 2005 to 2018 in Lang Son province. The estimated proportion of deaths due to breast cancer was 1.17\% (210 cases of breast cancer vs. 17,990 total cases) in women. \& Crude rate per 100,000 personyears; \$ Age-standardized rate per 100,000 person-years using the World Health Organization standard population for 2000-2025; \# Proportion of death cases aged under 70 year-olds. Missing data for 2009-2010.

\begin{tabular}{|c|c|c|c|c|}
\hline Year & Case & Crude rate \& & $\%<70 \#$ & WHO-ASR \$ \\
\hline 2005 & 19 & 5.3 & 73.7 & 5.9 \\
\hline 2006 & 11 & 3.0 & 81.8 & 3.2 \\
\hline 2007 & 14 & 3.8 & 71.4 & 4.1 \\
\hline 2008 & 16 & 4.3 & 81.3 & 5.0 \\
\hline 2011 & 16 & 4.3 & 62.5 & 4.9 \\
\hline 2012 & 24 & 6.4 & 87.5 & 7.0 \\
\hline 2013 & 12 & 3.2 & 75.0 & 3.9 \\
\hline 2014 & 17 & 4.3 & 94.1 & 4.7 \\
\hline 2015 & 20 & 5.1 & 85.0 & 5.8 \\
\hline 2016 & 18 & 4.6 & 77.8 & 4.9 \\
\hline 2017 & 21 & 5.3 & 95.2 & 6.1 \\
\hline 2018 & 22 & 5.6 & 81.8 & 6.4 \\
\hline
\end{tabular}

Table 3: Mortality rates ratio and 95\% confidence interval by years from 2005 to 2018 in Lang Son province.

\begin{tabular}{|c|c|c|c|}
\hline Year & Case & Crude MRR (95\%CI) & Age-adjusted MRR (95\%CI) \#\# \\
\hline $2005-2006$ & 30 & 1.00 (reference) & 1.00 (reference) \\
\hline 2007 & 14 & $0.92,(0.49,1.74)$ & $0.92,(0.49,1.74)$ \\
\hline 2008 & 16 & $1.04,(0.57,1.90)$ & $1.04,(0.57,1.90)$ \\
\hline 2011 & 16 & $1.06,(0.58,1.94)$ & $1.06,(0.58,1.94)$ \\
\hline 2012 & 24 & $1.55,(0.91,2.66)$ & $1.56,(0.91,2.66)$ \\
\hline 2013 & 12 & $0.79,(0.40,1.54)$ & $0.79,(0.40,1.54)$ \\
\hline 2014 & 17 & $1.05,(0.58,1.91)$ & $1.05,(0.58,1.91)$ \\
\hline 2015 & 20 & $1.23,(0.70,2.16)$ & $1.23,(0.70,2.16)$ \\
\hline 2016 & 18 & $1.11,(0.62,1.99)$ & $1.11,(0.62,1.99)$ \\
\hline 2017 & 21 & $1.28,(0.73,2.23)$ & $1.28,(0.73,2.23)$ \\
\hline 2018 & 22 & $1.36,(0.78,2.35)$ & $1.36,(0.78,2.35)$ \\
\hline$p$ for Trend & & 0.209 & 0.209 \\
\hline
\end{tabular}

Note: \#\# adjusted for age groups (0-9, 10-19, 20-29, 30-39, 40-49, 50-59, 60-69, 70-79, 80+). Missing data for 2009-2010.

\section{Discussion}

We observed a serious prelature breast cancer mortality in the Lang Son province, remote mountainous areas. Female breast cancer has presented at young age women and the proportion of premature death was very high, over four-fifth of total death cases. The result has highlighted the importance of public health policies for better prevention and management of breast cancer screening to reduce premature death in the study population. Moreover, financial support policies should be implemented to promote access to appropriate diagnosis and treatment for women living in disadvantaged areas. After adjusting with the World Health Organization standard population, 2000-2025, the ASR of breast cancer in Lang Son province is 5.2/100,000 during study time from 2005 to 2018. In consistence, the studies in Chinese women living 
in rural areas, the ASR in 2013 and 2014 of breast cancer were $5.59 / 100000$ and 5.79/100 000, respectively [6,12]. The ASR of breast cancer of our study is lower compared to one among women living in Ha Noi - the capital of Vietnam in the period from 19962005 and the ASR of breast cancer in Vietnam in 2012, which were as high as 13 and 9.9, respectively $[13,14]$. Hanoi City is the highest urbanization that can be explained why the breast cancer in this city was higher than in the Lang Son province.

To reduce young age death from breast cancer, the National Cancer Control Programs increasing the awareness of early detection of breast cancer and providing free screening for breast cancer and cervical cancer has been conducted in Vietnam since 2008. There were other several programs such as the "Early detection of breast cancer and cervical cancer in women" program implemented from 2012, the "We care for her" happening in 2013-2014. The Vietnam Women's Union has representatives at every commune has been also running programs to educate how to do breast self-examination [8]. Moreover, treatment for breast cancer in Vietnam has been remarkably improved recently. Bettertolerated therapies have been replacing ablative surgery and aggressive chemotherapy. Tamoxifen or other hormonal therapies, cytotoxic, and targeted therapies, shown to significantly reduce breast cancer recurrence and mortality in breast cancer patients $[15,16]$, are all available in Vietnam [8]. In addition, the population variation of our study comparing the previous studies might be likely the inducement. Nationwide, Vietnam has many cities in the progress of "modernization", where women are at increasingly high risk of breast cancer such as decreased parity, delayed childbirth, less breastfeeding which was demonstrated to be risk factors of breast cancer in low-income countries as well as in Vietnam $[17,18]$. However, these programs have been piloted at the areas of urbanization of the Hanoi and Ho Chi Minh Cities, but that might not be ready in the Lang Son province.

In terms of the trend mortality rates during the study period, there was a non-increasing trend during 2005-2018 but it is suggested to be increasing soon due to many environmental factors and lifestyles of an unhealthy diet, tobacco smoking, harmful usage of alcohol, and lack of physical activities. This result is consistent with previous studies showing the rise of breast cancer mortality in other territories. For instance, according to a global analysis, there was a significant increase in breast cancer mortality rates in all super regions. For total world countries, the mean breast cancer mortality rate was 13.77 per 100,000 in 1990 and the overall increasing slope of the mortality rate was 0.7 per 100,000 from 1990 to 2015 [3]. Another study presented a tendency of increased deaths from breast cancer in Brazilian women, particularly in young women from 1996 to 2013 [19]. In China, the standardized mortality rate of breast cancer was similarly shown to have an upward trend [20]. To improve cancer prevention for Vietnamese women, it is important to establish better public health policies and management of breast cancer, especially in remotes areas. It was reported that the majority of breast cancer patients in Vietnam are detected at the advanced stages [21], which was revealed to be the result of poor knowledge and awareness among the general public [22].

In a study conducted in the mountainous area in Northern Vietnam, the level of knowledge and practice about breast selfexamination, clinical breast examination, breast ultrasound, and mammography were still adverse. Approximately $17 \%$ of women mentioned clinical breast examination, and only 13.8\% reported practicing breast self-examination [23]. Among women living in one rural district, more than half of all the participants, including both younger (69.5\%) and older (53.3\%) women, believed that they would not get breast cancer if they took good care of themselves [24]. In central cities such as Hanoi and Ho Chi Minh City, the prevalence of sufficient knowledge and practice breast self-examination among female textile workers were only $22.7 \%$ and $15.8 \%$ [25]. In this context, increasing awareness about the importance of breast cancer screening and developing early detection strategies for breast cancer is essential. Community education programs and low-cost screening approaches such as clinical breast examination should be conducted widely to ensure the accessibility of all Vietnamese women, especially ones residing in remotes areas. The mammography screening should be suggested in high-risk women according to the age-specific mortality rate results.

Furthermore, the long treatment course of breast cancer has been causing a significant financial burden to the patients, especially the patients without health insurance. A recent study conducted in South East Asia, including Vietnam, showed that $48 \%$ of cancer patients incurred financial catastrophe within one year after the diagnosis [26]. It was estimated that the annual medical expenses for breast cancer treatment amounted to $18 \%$ of gross national income (GNI) per capita in Vietnam in 2010 (\$195 vs. $\$ 1,100$ ) [27]. These facts again emphasize the role of breast cancer screening in Vietnamese women since the diagnosis at the early stage can reduce significantly the cost of initial treatment. Besides, the financial burden of the treatment course could be a barrier to seeking care and to appropriate treatment compliance, which may contribute to the higher mortality of breast cancer. Therefore, establishing a policy of universal health insurance coverage along with other financial supports would improve access to medical care and the prognosis of breast cancer patients in Vietnam. The government should give financial support to breast cancer patients whose cost of illness exceeds their ability to pay. Furthermore, a 
network of primary health care such as home care and community care should be promoted to provide health care services to breast cancer women throughout the country. These settings may improve compliance with treatment and reduce costs for patients in Vietnam, where access to health facilities for cancer treatment has been limited. This combination would encourage the patients to comply with their long-term treatment and thus contribute to reducing deaths from cancers, including breast cancer.

Moreover, the current vertical and central organization of health care services in Vietnam may limit the accessibility of medical treatment for breast cancer patients living in mountainous areas. The lack of surgical oncologists, medical and radiation oncologists, anesthetists, and pathologists at the low level of the health system also has a significant effect on providing comprehensive treatment to breast cancer patients. Authorizing and supporting district and commune health stations with the appropriate health infrastructure and trained healthcare workforce could have a positive impact in terms of reducing in-direct costs for women with low - income to encourage them not to bypass them in favor of seeking suitable treatment as well as in reducing overcrowding in central and provincial hospitals. Breast cancer deaths in our study mostly occurred in reproductive-aged women, increasing rapidly from 40 years old, reaching a peak at the ages of 60-69, and then falling. This result is logical according to one study showing that the breast cancer incidence in Vietnamese women was highest at the age of 50 [21]. Meanwhile, the overall survival rate for breast cancer patients was $85.01 \pm 1.61$ months [28]. Similarly, according to a study investigating the breast cancer mortality rate in China during 1991 - 2011 showing that breast cancer usually occurs post-puberty, and its incidence increases slowly from 30 years old, reaching a peak at the ages of 40-60, and the first death peak occurring at 55 to 65 years [20]. In this sense, the clinical protocols and public policies that encourage early detection for breast cancer, and the key population should be among women between ages 40 to 65 . Breast cancer screening using clinical breast examination for women aged 40 to 55 years was identified to be very costeffective in Vietnam according to the World Health Organization criteria [29]. Another study demonstrated offering the first round of mammography screening to Vietnamese women aged 50-59 years was cost-effective, with the given threshold of three times the Vietnamese GDP per capita [30]. Therefore, breast cancer screening programs need to be provided to women aged 40-65 in the combination of clinical breast cancer and mammography to improve the life expectancy for breast cancer patients as well as reduce the public economic burden.

Several limitations should be noted in this study. Firstly, it was done in only one mountainous province in the North of Vietnam, so the findings may not be generalized to all Vietnamese women. Second, there was a deficit of the information system concerning factors associated with mortality from breast cancer that is restricted in the death record. This study, however, is the first investigation of the mortality rate of breast cancer in the province at a continuous long-term period. The findings presented here were similar to and different from those reported elsewhere. Overall, the results of this study will increase our understanding and guide interventions to improve early screening for breast cancer strategies specifically for Vietnamese women, especially in remote areas, with adverse socioeconomic status. Cancer mortality rates estimated in this study are reliable because there were no duplicate records. At each commune, the head of the commune Health station followed up carefully all fatal cases occurring at his commune while giving medical care and household visiting until the result as neighborhood relationship as well as a duty of the appointed medical worker position. Despite these limitations, the present findings had highlighted the public health problem of premature breast cancer in the low-medium economic countries that warrant global action plans against this preventable disease.

\section{Acknowledgment}

We express special thanks to the heads of commune health stations in Lang Son province for providing valuable data for the present study and his staff members in running the process of death registration for a long period of study.

\section{Funding}

There was no funding.

\section{References}

1. Torre LA, Islami F, Siegel RL, Ward EM, Jemal A (2017) Global Cancer in Women: Burden and Trends. Cancer epidemiology, biomarkers \& prevention: a publication of the American Association for Cancer Research, cosponsored by the American Society of Preventive Oncology 26(4): 444-457.

2. Bray F, Ferlay J, Soerjomataram I, Siegel RL, Torre LA, et al. (2018) Global cancer statistics 2018: GLOBOCAN estimates of incidence and mortality worldwide for 36 cancers in 185 countries. CA: a cancer journal for clinicians 68(6): 394-424.

3. Azamjah N, Soltan Zadeh Y, Zayeri F (2019) Global Trend of Breast Cancer Mortality Rate: A 25-Year Study. Asian Pacific journal of cancer prevention: APJCP 20(7): 2015-2020.

4. DeSantis CE, Ma J, Goding Sauer A, Newman LA, Jemal A (2017) Breast cancer statistics, 2017, racial disparity in mortality by state. CA: a cancer journal for clinicians 67(6): 439-448.

5. Fan L, Strasser Weippl K, Li JJ, St Louis J, Finkelstein DM, et al. (2014) Breast cancer in China. The Lancet Oncology 15(7): e279-289.

6. Zuo TT, Zheng RS, Zeng HM, Zhang SW, Chen WQ (2017) Female breast cancer incidence and mortality in China, 2013. Thoracic Cancer 8(3): 214-218. 
7. Pham T, Bui L, Kim G, Hoang D, Tran T, et al. (2019) Cancers in Vietnam-Burden and Control Efforts: A Narrative Scoping Review. Cancer control: journal of the Moffitt Cancer Center 26(1): 1073274819863802.

8. Jenkins C, Minh LN, Anh TT, Ngan TT (2018) Breast cancer services in Vietnam: a scoping review 11(1): 1435344.

9. Nguyen TT, Nguyen TT (2014) Cost-Utility Analysis of Trastuzumab in Treatment Of Metastatic Her ${ }_{2}$-Positive Breast Cancer in Vietnam. Value in health: the journal of the International Society for Pharmacoeconomics and Outcomes Research 17(7): A643.

10. Web Portal Lang Son Province.

11. Stevenson MR, Ngoan le T, Hung DV, Huong Tu NT, Mai AL, et al. (2012) Evaluation of the Vietnamese A6 mortality reporting system: injury as a cause of death. Injury prevention: journal of the International Society for Child and Adolescent Injury Prevention 18(6): 360-364.

12. Li H, Zheng RS, Zhang SW, Zeng HM, Sun XK, et al. (2018) Incidence and mortality of female breast cancer in China, 2014. Zhonghua Zhong Liu Za Zhi Chinese journal of oncology 40(3): 166-171.

13. Ngoan le T (2006) Cancer mortality in a Hanoi population, Vietnam, 1996-2005. Asian Pacific journal of cancer prevention: APJCP 7(1): 127 130.

14. Ghoncheh M, Mohammadian Hafshejani A, Salehiniya H (2015) Incidence and Mortality of Breast Cancer and their Relationship to Development in Asia. Asian Pacific journal of cancer prevention: APJCP 16(14): 6081-6087.

15. Burton R, Stevenson C (2020) Assessment of Breast Cancer Mortality Trends Associated With Mammographic Screening and Adjuvant Therapy From 1986 to 2013 in the State of Victoria, Australia. JAMA network open 3(6): e208249.

16. Vilaprinyo E, Puig T, Rue M (2012) Contribution of early detection and adjuvant treatments to breast cancer mortality reduction in Catalonia, Spain. PloS one 7(1): e30157.

17. Porter P (2008) Westernizing women's risks? Breast cancer in lower-income countries. The New England journal of medicine 358(3): 213-216.

18. Trieu PDY, Mello Thoms C, Peat JK, Do TD, Brennan PC (2017) Risk Factors of Female Breast Cancer in Vietnam: A Case-Control Study. Cancer research and treatment: official journal of Korean Cancer Association 49(4): 990-1000.

\section{ISSN: 2574-1241}

DOI: $10.26717 /$ BJSTR.2022.40.006525

Kim Cat Tuyen Vo. Biomed J Sci \& Tech Res

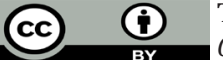

This work is licensed under Creative Commons Attribution 4.0 License

Submission Link: https://biomedres.us/submit-manuscript.php
19. Rocha Brischiliari SC, Oliveira RR, Andrade L, Brischiliari A, Gravena AA, et al. (2017) The Rise in Mortality from Breast Cancer in Young Women: Trend Analysis in Brazil. PloS one 12(1): e0168950.

20. Shi XJ, Au WW, Wu KS, Chen LX, Lin K (2014) Mortality characteristics and prediction of female breast cancer in China from 1991 to 2011. Asian Pacific journal of cancer prevention: APJCP 15(6): 2785-2791.

21. Trieu PD, Mello Thoms C, Brennan PC (2015) Female breast cancer in Vietnam: a comparison across Asian specific regions. Cancer biology \& medicine 12(3): 238-245.

22. Anh PT, Duc NB (2002) The situation with cancer control in Vietnam. Japanese journal of clinical oncology 32: S92-s97.

23. Toan DTT, Son DT, Hung LX, Minh LN, Mai DL, et al. (2019) Knowledge, Attitude, and Practice Regarding Breast Cancer Early Detection Among Women in a Mountainous Area in Northern Vietnam. Cancer control: journal of the Moffitt Cancer Center 26(1): 1073274819863777.

24. Kim JG, Hong HC (2019) Cultural beliefs about breast cancer in Vietnamese women 19(1): 74 .

25. Tuyen DQ Dung TV (2019) Breast Self-Examination: Knowledge and Practice Among Female Textile Workers in Vietnam 26(1): 1073274819862788 .

26. Kimman M, Jan S, Yip CH, Thabrany H, Peters SA, et al. (2015) Catastrophic health expenditure and 12 -month mortality associated with cancer in Southeast Asia: results from a longitudinal study in eight countries. BMC medicine 13: 190 .

27. Hoang Lan N, Laohasiriwong W, Stewart JF, Tung ND, Coyte PC (2013) Cost of treatment for breast cancer in central Vietnam. Global health action 6: 18872.

28. Nguyen VC, Nguyen TQ, Vu TNH, Phung TH, Nguyen TPH, et al. (2019) Application of St Gallen Categories in Predicting Survival for Patients With Breast Cancer in Vietnam. Cancer control: journal of the Moffitt Cancer Center 26(1): 1073274819862794

29. Nguyen LH, Laohasiriwong W, Stewart JF, Wright P, Nguyen YTB, et al. (2013) Cost-Effectiveness Analysis of a Screening Program for Breast Cancer in Vietnam. Value in health regional issues 2(1): 21-28.

30. Nguyen CP, Adang EMM (2018) Cost-effectiveness of breast cancer screening using mammography in Vietnamese women. PloS one 13(3): e0194996.

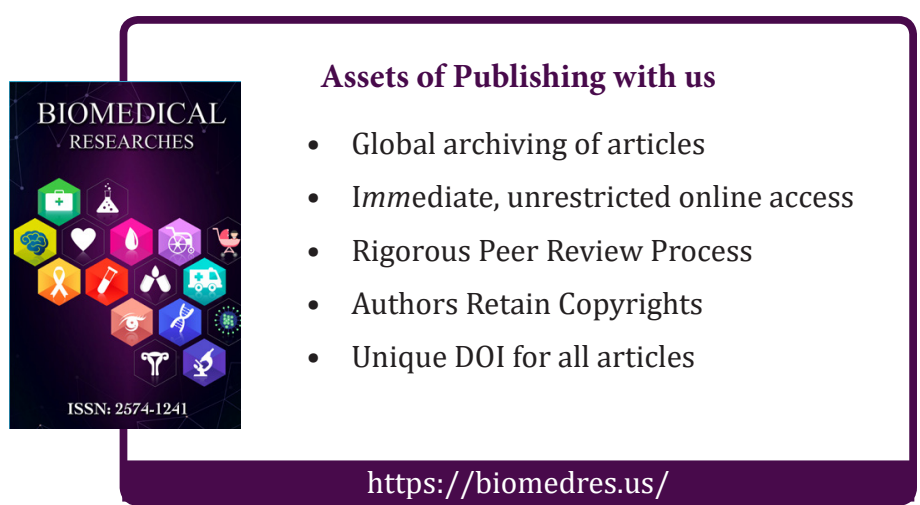

